(C) [2007] IEEE. Reprinted, with permission, from [S. Kodagoda, S. Sehestedt, A. Alempijevic, Z. Zhang, A. Donikian and G. Dissanayake, Towards an Enhanced Driver Situation Awareness System, Industrial and Information Systems, 2007. ICIIS 2007. International Conference on, 9-11 Aug. 2007]. This material is posted here with permission of the IEEE. Such permission of the IEEE does not in any way imply IEEE endorsement of any of the University of Technology, Sydney's products or services. Internal or personal use of this material is permitted. However, permission to reprint/republish this material for advertising or promotional purposes or for creating new collective works for resale or redistribution must be obtained from the IEEE by writing to pubs-permissions@ieee.org. By choosing to view this document, you agree to all provisions of the copyright laws protecting it 


\title{
Towards an Enhanced Driver Situation Awareness System
}

\author{
S. Kodagoda, S. Sehestedt, A. Alempijevic, Z. Zhang, A. Donikian and G. Dissanayake \\ ${ }^{1}$ ARC Centre of Excellence for Autonomous Systems, Faculty of Engineering, University of Technology, Sydney NSW \\ Australia \\ s.kodagoda, s.sehestedt, a.alempijevic, z.zhang, a.donikian, g.dissanayake@cas.edu.au
}

\begin{abstract}
This paper outlines our current research agenda to achieve enhanced driver situation awareness. A novel approach that incorporates information gathered from sensors mounted on the neighboring vehicles, in the road infrastructure as well as onboard sensory information is proposed. A solution to the fundamental issue of registering data into a common reference frame when the relative locations of the sensors themselves are changing is outlined. A description of the vehicle test bed, experimental results from information gathered from various onboard sensors, and preliminary results from the sensor registration algorithm are presented.
\end{abstract}

\section{INTRODUCTION}

Road safety is a worldwide concern [1]. Developed countries have managed to significantly reduce the impact of road traffic injuries/fatalities by developing infrastructure, enforcing legislation to control speed and alcohol consumption, mandating the use of seatbelts and crash helmets. A comprehensive five year study of road accidents by Treat et al [2] found that the human error was the sole factor in $57 \%$ and a contributing factor in $92.6 \%$. They also concluded that over $80 \%$ of all automobile accidents are due to human perceptual error. Therefore, further reduction of road related accidents can be achieved by enhancing driver perception by means of intelligent sensing and advanced processing.

Our approach of enhancing road safety is by utilizing information about the vehicle internal status (dynamics), status of the surrounding environment and the driver functional status (Fig. 1). The vehicle dynamics are estimated by a Kalman filter based algorithm with observations from an on-board Global Positioning System (GPS) and an Inertial Measurement Unit (IMU). The environment perception is achieved by processing of the on-board sensory data (cameras, lasers and a radar) as well as the sensory data from neighboring vehicles and the road infrastructure. This facilitates an enhanced field of view. With the developments in inter vehicular communications [3] and roadway intelligent infrastructure [4-5], it is likely that transmitting data in real time between infrastructure and neighboring vehicles will become feasible in the near future. Therefore, feasibility of incorporating such sensory signals in driver situation awareness systems is closer than ever before. Furthermore, it is our intention to estimate human factors such as fatigue, sleepiness and driver behaviors in real-time for enhancing road safety.

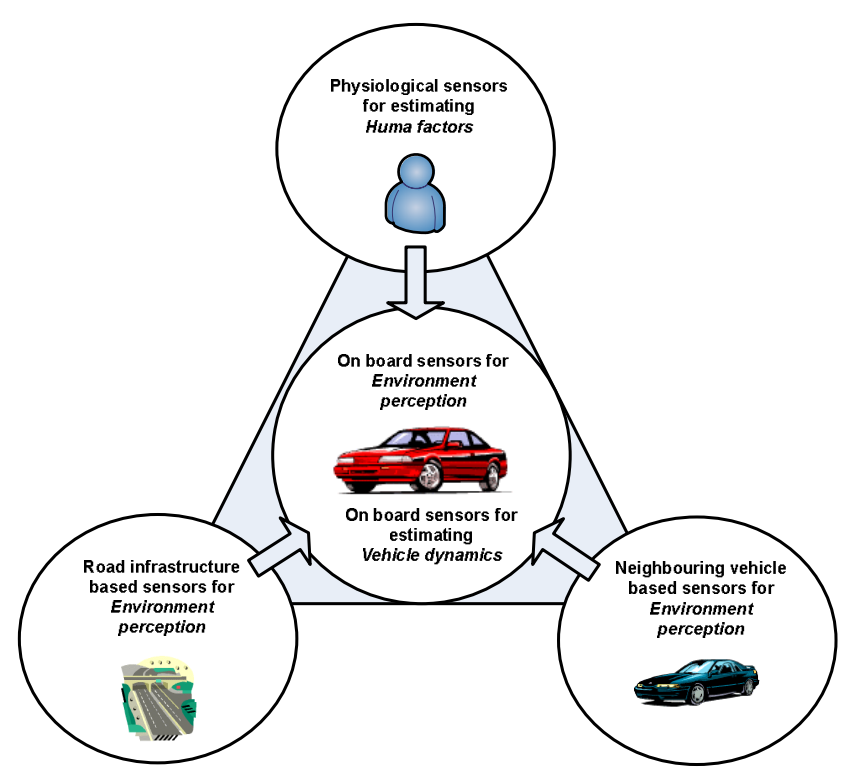

Fig. 1 An integrated solution for road safety

\section{DEVELOPMENT OF THE CRUISE}

The CAS Research Ute for Intelligence, Safety and Exploration (CRUISE) is a Ford courier fitted with variety of sensors and computing hardware (Fig.2).
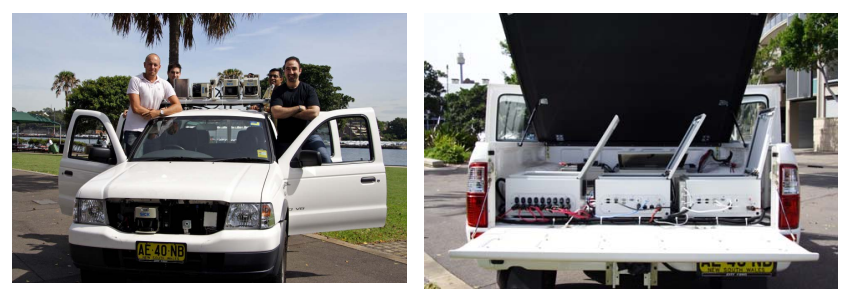

Fig. 2 CRUISE test-bed

\section{A Hardware Architecture}

Two computers are used to process data from a Global Positioning System (GPS), Inertial Measurement Unit (IMU), two accelerometers, four Laser Measurement Systems (LMS) and two cameras (Fig. 3). We are in the process of integrating a millimeter wave radar, a three dimensional vision system, electroencephalogram(EEG), electromyogram(EMG) and respiratory sensors, which will provide us with comprehensive information on the status of 
the vehicle, driver and the environment. All the computers are synchronized via the local area network.

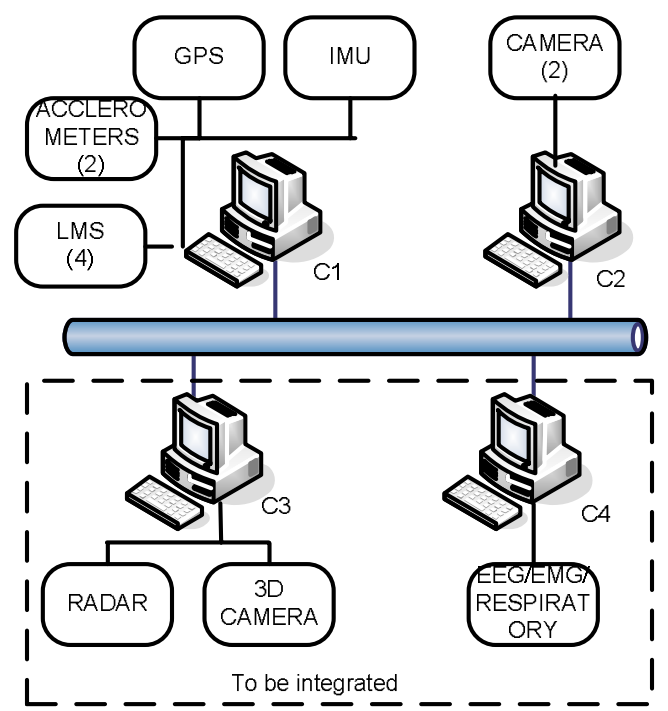

Fig. 3 Hardware architecture

\section{B Software Architecture}

The operating system used for all the on-board computers is Linux (Kubuntu), except for C4. Windows XP is used for this computer due to the unavailability of Linux drivers for the EEG/EMG/Respiratory system. The software uses a Component-Based Software Engineering (CBSE) approach. This offers modularity, software reuse, and flexibility in deployment. Components run asynchronously and exchange information through communication.

ZeroC's Ice middleware [6] is used extensively in the system: for component interface definition, intercomponent communication, component deployment, location, activation services, etc. Orca [7], which is an open source project that customizes Ice to robotic applications, is used. Orca provides an on-line repository of reusable components.

\section{ON-BOARD SENSORS FOR ESTIMATION OF VEHICLE DYNAMICS}

The vehicle dynamic parameters are critical for analyzing safety of a vehicle. Sensors used for estimating the vehicle dynamics are a Novatel Propac G2+ GPS system [8], Crossbow's IMU700 CA inertial measurement unit [9] and, two Jewell LCF $200-2 \mathrm{G}$ servo accelerometers. The GPS provides position solutions at $1 \mathrm{~Hz}$. The IMU provides raw acceleration and rotational data in three orthogonal axes at $160 \mathrm{~Hz}$. The servo accelerometers are used to determine the initial bank and elevation of the platform. The "Direct feedback" [10] filtering method is used for estimating the vehicle dynamic parameters. As shown in Fig. 4, the Inertial Navigation Solution (INS) provided by the integration of the fast IMU data is compared with external GPS observations to estimate the biases and drifts. The states of the estimator are observation errors of INS and GPS positions and velocities. In this structure the estimated errors of position, velocities and attitudes are fed back to the INS, thus minimizing the growth of the observed error that is delivered as an observation to the filter.

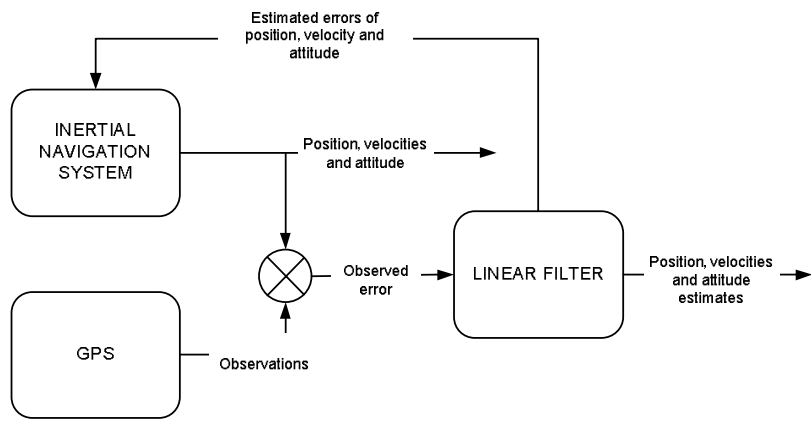

Fig. 4 The direct feedback filter [10]

We have carried out experiments to assess the filter performance. The vehicle is driven along a straight road at about $40 \mathrm{~km} / \mathrm{h}$ while giving impulse steering inputs at constant intervals. Some of the outputs of the estimator are shown in Fig. 5.

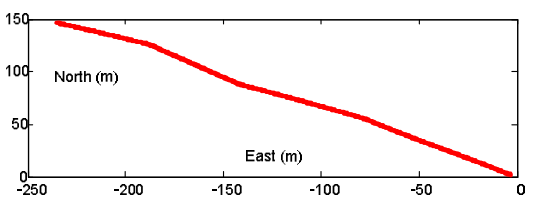

(a) vehicle trajectory
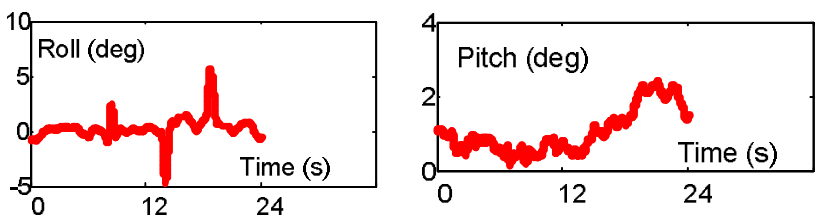

(b) roll angle

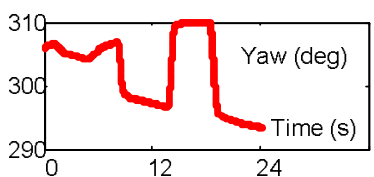

(c) pitch angle

(d) yaw angle

(e) slip angle

Fig. 5 Some of the estimated states of the vehicle

\section{ON-BOARD SENSORS FOR ENVIRONMENT PERCEPTION}

A comprehensive environment perception is vital in avoiding accidents. The perception sensors currently mounted on the CRUISE are a forward looking roof mounted camera and a front bumper bar mounted laser range finder (LMS) for road/obstacle detection, two roof mounted LMSs for mapping the ground for generating the traversability map. One LMS is mounted on the back of the CRUISE for rear view. A millimeter wave radar and a three dimensional camera are currently being integrated to the 
sensor suite. Work so far has focused on solving road boundary and pedestrian detection problems.

Conventional approach to road boundary detection problem is to use a generic algorithm to solve all the possible scenarios in a road environment. Due to the complex nature of the roads, such generic methodologies tend to have high failure rate. Our approach is to use a combination of algorithms tuned for a several set of scenarios to achieve complex road boundary detection.

\section{A Lane marking detection and tracking}

The development of situation awareness systems and autonomous driving requires the ability to analyze the road scene. One prerequisite for this is the extraction of lanes and lane markings. This information is essential in order to obey traffic rules and to detect possible hazards. This work focuses on a novel approach to robustly extract lane markings in urban traffic scenarios. Much of the work presented in the literature concentrate on highway like scenarios, where the traffic situations are less complex and markings are clearly visible. Assuming geometric models of roads, such as lanes being straight or curved is only appropriate for freeways but is certain to fail in urban areas. The main reasons being the complexity of roads, bad visibility of the markings, occlusions or missing markings over extended periods of time. Therefore, strong assumptions about the lane geometry can lead to failure and hence weaker models are preferable.

Low level image processing for lane marking detection is performed on the remapped image (IPM) applying a lane model which stipulates that a road marking is represented by a predominantly bright line (lane marking) of constant width surrounded by a darker region (the road). Thus, the pixels belonging to a road marking have a brightness value higher than their left and right neighbours at a given horizontal distance. A vertical edge in an image conforms similarly to the same principle, however the intensity difference between neighbouring pixels must be over a threshold to be validated as a lane marking. Therefore, an exhaustive search across each row of the image will produce potential lane marking candidates where the match probability can be measured with the edge quality.

Then we use a particle filter based approach, where particles are used to represent a possible position of a piece of lane marking, including a weight that is proportional to the probability that a lane marking is intact present. Particles are initialized at the bottom of the image and move to the top seeking lane markings [11]. The outcome of the lane detection algorithm in the presence of shadows, other vehicles and at a bend are shown in Fig. 6 .

\section{B Road boundary detection}

Roads without lane markings or dirt roads are not uncommon in most of the road networks. In such scenarios, the lane marking detection algorithm does not provide any meaningful results. Therefore, we have developed another vision based algorithm for detecting unmarked or dirt roads. The algorithm is based on the work by Bradski, et al [12]. The rationale is to use information from the laser range finder to identify drivable regions in the environment and assuming it to be a possible road, a region of interest (ROI) is defined and transformed onto the image plane. Such an image window is considered as a model of the road and is used in a classification algorithm to extend the image window to the whole road segment in the image. In [12], RGB channels are used in defining the road model. We have noticed that RGB implementation has poor performance when shadows are present. We are currently investigating other color spaces such as HSV and C1, C2, C3. Texture of an image is another property that can be used to describe the ROI. Image sequence in Fig. 7 and 8 show the detection of the boundaries of a dirt road and a road without lane markings.
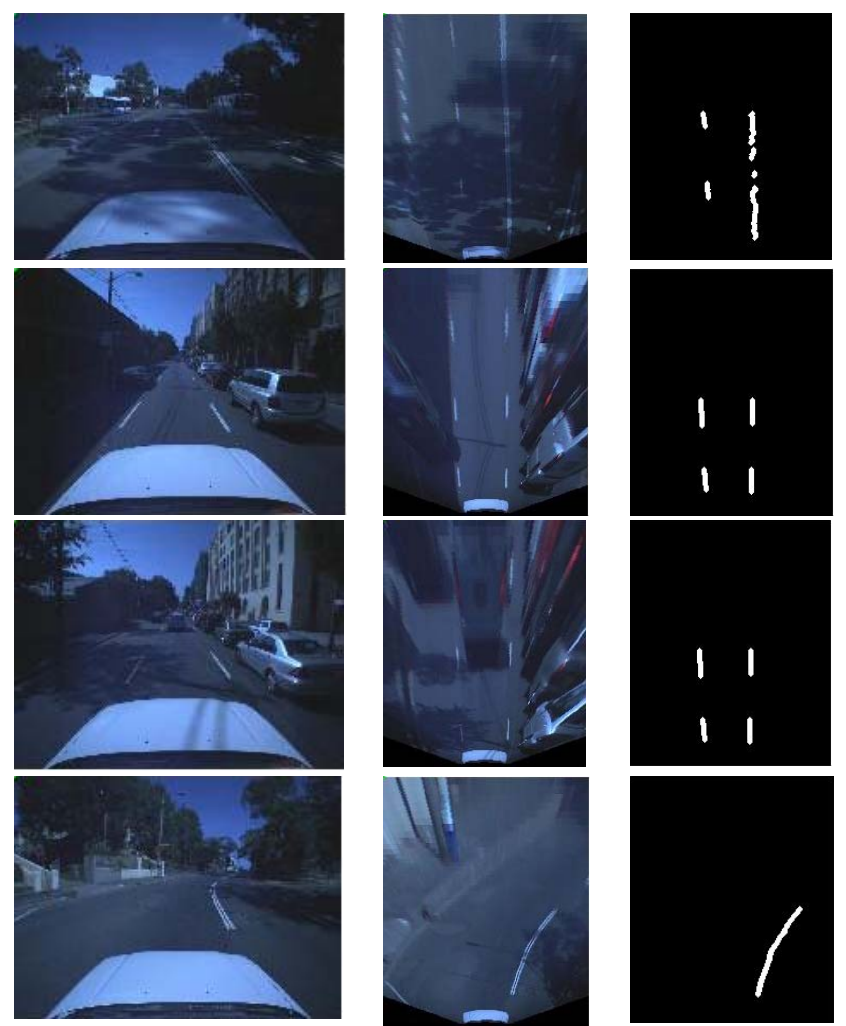

Fig. 6 Results of particle filter based lane detection: left most column original images, middle column - IPM images, right most column detected lane markings

\section{Pedestrian detection}

Pedestrian detection is an important part in improving road safety, especially in urban roads. Generally, people detection is carried out by processing a monocular camera image. One of the methods is to use a template matching technique by scanning the whole image to find a match [13] which incurs a high computational cost. The solution is further complicated by the presence of mismatches in scale between the template and the image requiring further computationally expensive steps to correct for this. We have used information from a laser rangefinder collocated with a camera to alleviate these problems effectively and efficiently [14]. The laser range finder can be used for detecting moving objects in the vicinity, and to define a Region of Interest (ROI) in the image. Then the template 
only needs to be correlated with that ROI, saving computation time. Furthermore, the laser range finder also provides with distance to the object, which can be used for resizing the ROI to the correct scale, saving database size and computation. Block diagram of the algorithm is given in Fig. 9.

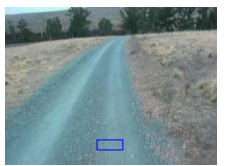

(a)

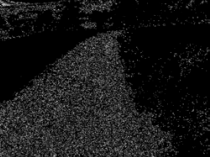

(b)

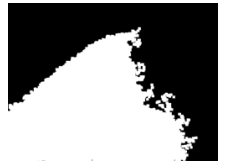

(c)
Fig. 7 Dirt road detection: (a) Original image, (b) Probability map based on the compatibility of the road model with individual pixels, (c) Segmented road

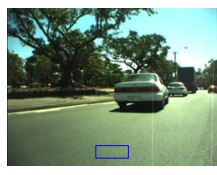

(a)

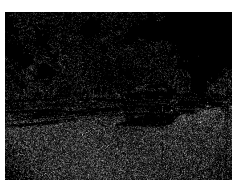

(b)

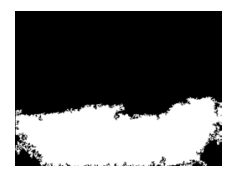

(c)
Fig. 8 Detection of roads with no lane markings: (a) Original image, (b) Probability map based on the compatibility of the road model with individual pixels, (c) Segmented road

First the laser data obtained at successive time steps (time ' $k$ ' and ' $k+1$ ') are used in an Iterative Closest Point (ICP) [15] algorithm to determine the change in the pose of the laser/vision system to detect moving objects. Then the regions containing moving objects detected using the laser data are further analyzed to remove the background. They are then mapped on to the image plane. The ROI is then resized using the distance to the moving objects obtained from laser scanner to match the scale of the templates in the database. The resized ROI is then compared with the templates to find the best match.

Pedestrian detection results are shown in Fig. 10. It is to be noted that pedestrians on the rightmost of the image can not be detected as possible ROIs are occluded by the vehicle on the right.

\section{$\mathrm{V}$ INFRASTRUCTURE BASED AND OTHER VEHICLE BASED SENSORS FOR ENHANCED PERCEPTION}

In improving driver situation awareness, it is very important to have a cocoon of sensors around the vehicle; however, occlusions can limit the sensing field of view. This effect is significant at intersections. Fortunately, the limited perception achievable by on-board sensors can significantly be improved by utilizing the sensory information of other sources such as infrastructure based sensors and information from neighboring vehicles. With the developments in inter vehicular communications [3] and roadway intelligent infrastructure [4-5], it will be feasible to transmit data in real time between infrastructure and neighboring vehicles. Therefore, feasibility of incorporating such sensory signals in driver situation awareness systems is closer than ever before. However, this inherently introduces a major challenge: un-calibrated data registration among moving observers.

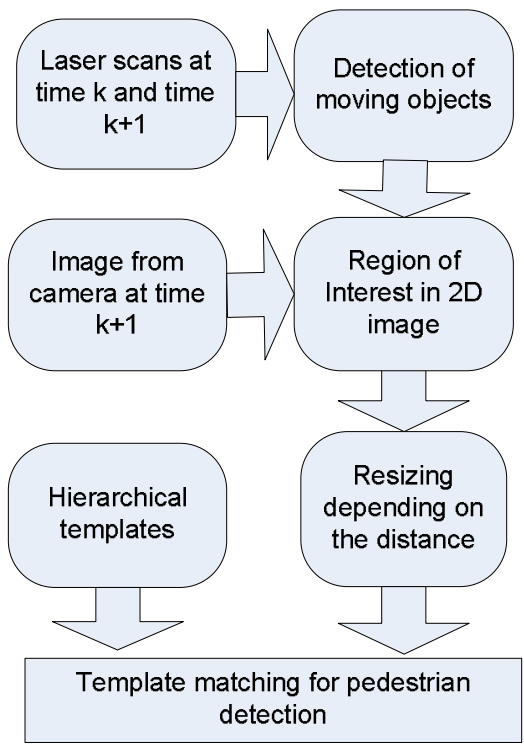

Fig. 9 The pedestrian detection algorithm
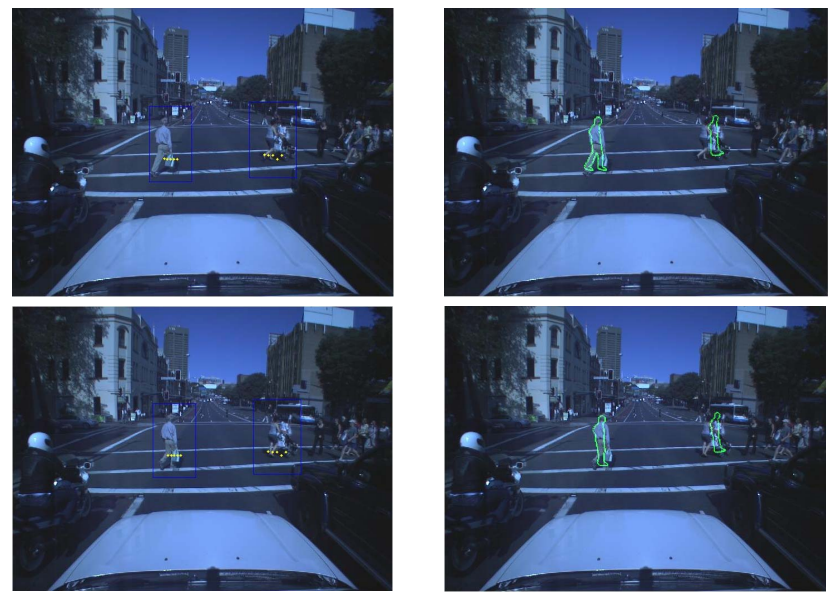

Left column: Original image showing mapped laser data (yellow) and detected ROI (blue rectangle)

overlaid in green

Fig. 10 Pedestrian detection results

Here, we exploited the principles of information theory and the efficiency of Fishers [16] implementation to compute mutual information to achieve registration and calibration of moving sensors observing dynamic objects in a scene. We do not assume any prior knowledge of relative location between the observers. Moving targets provide very little instant information in signal level. However, tracked dynamic targets provide substantial amount of information in the feature level. Therefore, we use feature level registration and select target attributes giving due regards to sensor modality and the rate of change of the attribute, as it is important that at least one attribute of the 
target exhibits a rate of change in both sensory spaces. Computing the MI then allows the registration of the common feature attributes in two sensory signals providing the relative location of the sensors. However. as the sensor itself is moving, the target attributes calculated on the sensory spaces may not be correlated. This issue can be solved by tracking the attributes of the targets in a locally defined world coordinate system and using the resulting feature within the MI framework. The target tracking problem is also complex due to the moving nature of the observer, maneuvering dynamics and occlusions. Multiple model approach, such as the Interacting Multiple Model (IMM) [17], provides one of the most effective frameworks for tracking maneuvering targets. False tracks that get initiated due to anomalies in the background and spurious data are eliminated using the Sequential Probability Ratio Test (SPRT) [18].

Fig 11 (a) shows a simulation study carried out with two moving observers ("O1" and "O2") and three maneuvering targets ("T1", "T2" and "T3"). Fig. 11(b) shows the extracted target attributes in respective locally defined world coordinates relative to the observers. The target attributes considered in this study are the range and bearing from the observer to the target and their speeds. The MI based algorithm converges at 39th iteration as depicted in Fig. 11(c), detecting that the speed of Target 2 is the most informative (Table I). The path of Target 2 in the two local world co-ordinate frames is then used to compute the relative calibration between the sensors through a least square fit. The tracked target poses in observer 2 can now be transformed onto the locally defined world reference frame of observer 1 (Fig 11(d)). It is to be noted that the target tracks from both observers overlap, confirming correct sensor registration.

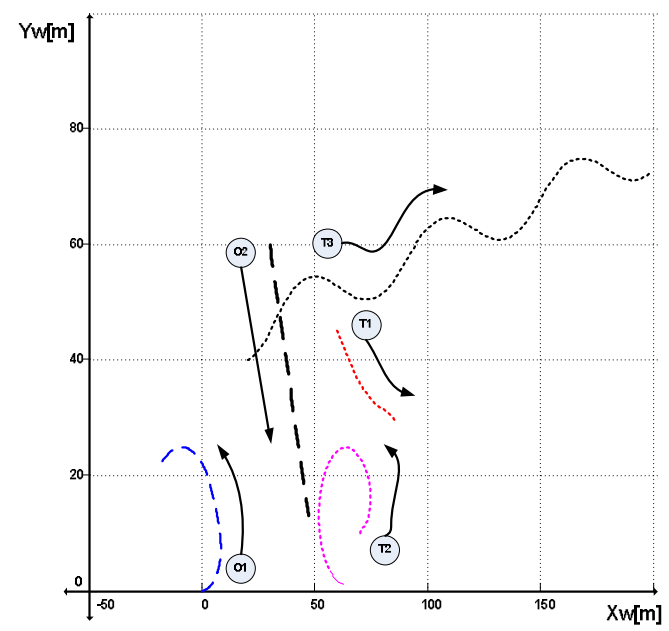

a) Simulation environment: $\mathrm{O}$ - observer, $\mathrm{T}$ - target

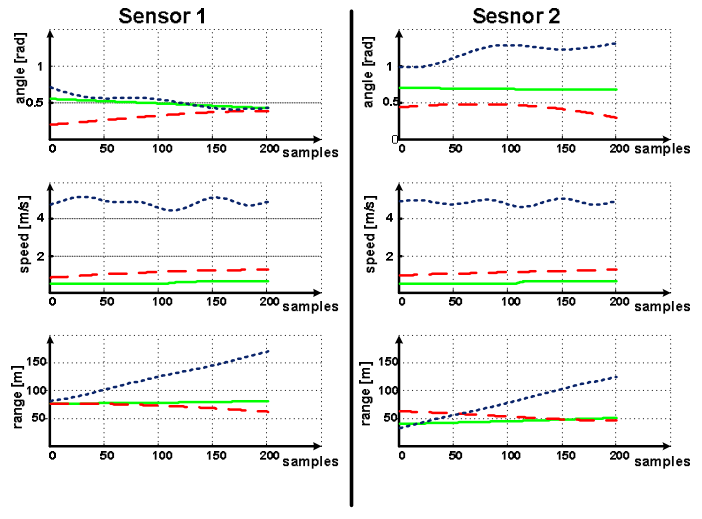

b) Extracted target attributes, solid line - target 1, dashed line - target 2, dotted line - target 3

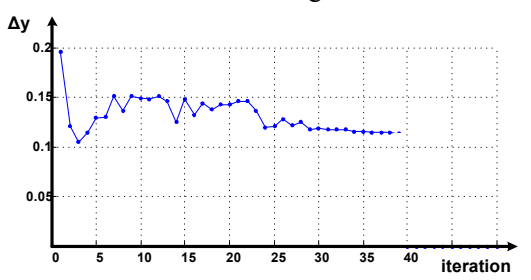

(c) learning curve

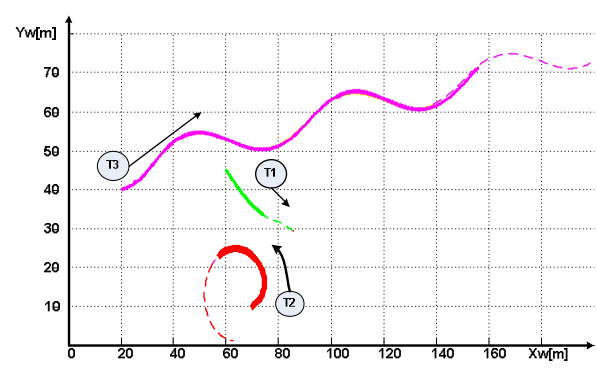

(d) All data transformed onto sensor 1 world reference frame

Fig 11 - Sensor registration with moving observers and maneuvering targets

TABLE I

MI values. a - angle, $\mathrm{s}-$ speed, $\mathrm{r}-$ range, $\mathrm{T}$ - target

\begin{tabular}{|c|c|c|c|c|c|c|c|c|c|}
\hline $\begin{array}{c}\text { Sensor/ } \\
\text { signal }\end{array}$ & $\begin{array}{c}\mathrm{a} . \\
\mathrm{T} 1 \\
\end{array}$ & $\begin{array}{c}\text { a. } \\
\text { T2 }\end{array}$ & $\begin{array}{c}\text { a. } \\
\text { T3 }\end{array}$ & $\begin{array}{c}\text { s. } \\
\text { T1 }\end{array}$ & $\begin{array}{c}\mathrm{s} . \\
\mathrm{T} 2\end{array}$ & $\begin{array}{c}\text { s. } \\
\text { T3 }\end{array}$ & $\begin{array}{c}\mathrm{r} . \\
\mathrm{T} 1\end{array}$ & $\begin{array}{c}\mathrm{r} . \\
\mathrm{T} 2\end{array}$ & $\begin{array}{c}\text { r. } \\
\text { T3 }\end{array}$ \\
\hline 1 & 0.9 & 50 & 15 & 0.06 & $78^{*}$ & 0.2 & 0.1 & 0.2 & 0.1 \\
\hline 2 & 19.6 & 22 & 3.8 & 1.9 & $100^{*}$ & 0.5 & 1.5 & 1 & 0.9 \\
\hline
\end{tabular}

\section{CONCLUSIONS}

In this paper, we described our ongoing work on enhanced driver situation awareness system. We proposed to utilize the onboard sensory information with information from vehicles in the vicinity and infrastructure while also considering human factors. Our current experimental results on this direction have been presented to demonstrate current achievements.

Our future work includes the integration of millimeter wave radar and PMD technologies three-dimensional camera for enhanced perception. We are also in the process of incorporating the EEG system for monitoring the human factors. 


\section{ACKNOWLEDGMENT}

This work is supported by the ARC Centre of Excellence program, funded by the Australian Research Council (ARC) and the New South Wales State Government. The authors also gratefully acknowledge the partial funding of this work by the German Academic Exchange Service (DAAD) with a PhD student scholarship.

\section{REFERENCES}

[1] World report on Road Traffic Injury Prevention: Summary, World Health Organization, Geneva, 2004

[2] Treat, J. R., Tumbas, N. S., McDonald, S .T., Shinar, D., Hume, R. D., Mayer, R. E., Stanisfer, R. L., and Castillan, N. J. (1977) Trilevel study of the causes of traffic accidents. Report No. DOTHS034-3-535-77, Indiana University.

[3] Tank T. and Linnartz J.M.G., "Vehicle to vehicle communications for AVCS platooning", IEEE Trans. Veh. Technol., vol. 46, pp. 528-536, May 1997.

[4] Kenward, M., "Intelligent Moves", The IEE Review, Vol.52, No.2, Feb. 2006, pp.22-23.

[5] Bishop, R., "Arizona I-19 Wi-Fi Corridor: Assessment of Opportunities for Probe Data perations", Report TRQS-02, October 2005,http:/www.azdot.gov/TPD/ATRC/publications/QuickStudies/ TRQS-02.pdf

[6] http://zeroc.com/

[7] http://orca-robotics.sourceforge.net/

[8] http://www.novatel.com/products/propakg2plus.htm

[9] http://www.xbow.com/Products/productdetails.aspx? sid=210
[10] Sukkarieh, S., "Low cost, high integrity, aided inertial navigation systems for autonomous land vehicles", $\mathrm{PhD}$ thesis, Australian centre for field robotics, University of Sydney, Australia.

[11] Stephan Sehestedt, Sarath Kodagoda, Alen Alempijevic and Gamini Dissanayake, "Robust Lane Detection in Urban Environments", in press, the IEEE/RSJ Int. Conf. on Intelligent Robots and Systems (IROS 2007), Oct., 2007.

[12] Garry Bradski, Adrian Kaehler and Vadim Pisarevsky, LearningBased computer vision with Intel's open source computer vision library, Intel Technology Journal, Vol.9, Issue. 2, May 2005, pp.119-130.

[13] D. M. Gavrila, "Multi-feature Hierarchical Template Matching using Distance Transforms", Proceedings of Pattern Recognition $14^{\text {th }}$ International Conference. vol. 1, 16-20 pp. 439-444, 1998.

[14] Zhengzhi Zhang and K. R. S. Kodagoda, "Multi-sensor Approach for People Detection", Proc. of the International Conference on Intelligent Sensors, Sensor Networks and Information Processing., February, 2005,(ISSNIP 2005), Melbourne, Australia, pp.355-36.

[15] A. F. Lu, E. Milios, "Robot pose estimation in unknown environments by matching 2D range scans", Journal of Intelligent and Robotic Systems, vol. 18, pp.249-275, 1997.

[16] J. W. Fisher, III and J. C. Principe, "Entropy manipulation of arbitrary nonlinear mappings," Proc. IEEE Neural Networks for Signal Processing, 1997.

[17] H. A. P. Blom and Y. Bar-Shalom, "The interacting multiple model algorithm for systems with Markovian switching coefficients", IEEE Trans. Automat. Contr., Vol.33, No. 8, Aug. 1988, pp.780783

[18] S. Blackman and R. Popoli, Design and Analysis of Modern Tracking Systems, Boston, Artech House, 1999. 